\title{
Multiple nonmelanocytic skin cancers in multiple regions
}

\author{
Song Hyun Han, \\ Soon Heum Kim, \\ Cheol Keun Kim, \\ Dong In Jo \\ Department of Plastic and \\ Reconstructive Surgery, Konkuk \\ University Chungju Hospital, Konkuk \\ University School of Medicine, Chungju, \\ Korea
}

The most common forms of nonmelanocytic skin cancer (NMSC) are basal cell carcinoma (BCC) and squamous cell carcinoma (SCC). The growing incidence of skin cancer in the Republic of Korea has sparked increasing scientific interest in these types of tumors. In the case described herein, multiple NMSCs occurred asynchronously in various areas of the body in a single patient. A 67-year-old man presented with an ulcerative lesion on the right retro-auricle and multiple keratinized masses on the back and face. The right retro-auricular lesion was diagnosed as BCC, and the keratinized masses on the patient's back and face were diagnosed as SCC. He subsequently presented with numerous pigmented skin lesions on the forehead, temple, pre-auricle, neck, right forearm, right hand, and both thighs. One lesion on the neck was diagnosed as BCC, and five lesions on the right hand and forearm were diagnosed as SCC. The patient was also diagnosed with supraglottic SCC and external auditory canal SCC. An otolaryngologist performed radical excision of the primary SCC. Suspected skin cancer lesions observed on the face and both ears were diagnosed as SCC. Patients with multiple NMSCs are at an elevated risk for additional skin cancers, making periodic follow-up important; furthermore, all suspicious lesions should be biopsied.

Keywords: Carcinoma in situ / Carcinoma, basal cell / Carcinoma, squamous cell / Skin neoplasms

\section{INTRODUCTION}

The two major subtypes of nonmelanocytic skin cancer (NMSC) are squamous cell carcinoma (SCC) and basal cell carcinoma (BCC). BCC is the most common form of skin cancer, followed by cutaneous squamous cell carcinoma (CSCC), which develops from the malignant proliferation of epidermal keratinocytes [1,2]. The head and neck area, which is frequently exposed to sunlight, accounts for $80 \%$ to $90 \%$ of SCCs and for most cases of BCC [3,4]. Most patients have only one form of skin cancer, but previous studies have demonstrated that pa-

Correspondence: Dong In Jo

Department of Plastic and Reconstructive Surgery, Konkuk University Chungju Hospital, Konkuk University School of Medicine, 82 Gugwon-daero, Chungju 27376, Korea

E-mail: cozarmd@kku.ac.kr

Received February 24, 2020 / Revised March 21, 2020 / Accepted April 27, 2020 tients are at an increased risk of developing additional SCC after the first occurrence $[5,6]$.

Although NMSC is common in Caucasians, an annual report of cancer statistics in the Republic of Korea published by the National Cancer Center documented 5,624 cases of newly diagnosed NMSCs in 2016, and stated that this number is steadily growing, with concomitant increases in medical costs. These trends have stimulated scientific interest in NMSC.

Herein, we report a rare case in which a patient was diagnosed with asynchronous multiple NMSCs on the head and neck, including the supraglottis and external auditory canal, as well as numerous other regions throughout the body.

\section{CASE REPORT}

A 67-year-old man presented to the hospital with an ulcerative 
lesion on the right retro-auricle and multiple keratinized masses on the back and face. His medical history included hypertension and SCC in situ on the dorsum of the right hand, which had been treated with a wide excision and an adipofascial flap at another hospital 5 years previously. He had no other diseases and no history of human papillomavirus (HPV) infection, but he was a farmer by profession and had a lifelong history of sunlight exposure. A $5 \times 5.5 \mathrm{~cm}$ area of diffuse skin discoloration was observed on the patient's back, along with several protruding masses. First, local excision of the protruding masses was performed. A histopathological examination confirmed these masses to be SCC in situ with a positive resection margin. Subsequently, wide excision of the lesion and a split-thickness skin graft (STSG) were performed (Fig. 1). In the right retro-auricular region, a $1.5 \times 1.5 \mathrm{~cm}$ ulcerative lesion was excised, and a histopathological examination confirmed that the lesion was BCC, with involvement of all the resection margins. Wide excision was performed, and the defect was covered using an STSG. The skin grafts took well, and the wounds healed very well (Fig. 2). A $1 \times 1 \mathrm{~cm}$ yellowish keratinized mass was observed on the right cheek. The mass was excised and the biopsy results revealed SCC in situ with a clean margin. The wound healed well, with no complications.

Eight months later, the patient presented with numerous pigmented skin lesions on the forehead, temple, pre-auricle, neck, right forearm, right hand, and both thighs. Twenty-three lesions were excised and biopsied. Primary closure was performed for small defects, while large defects were covered with
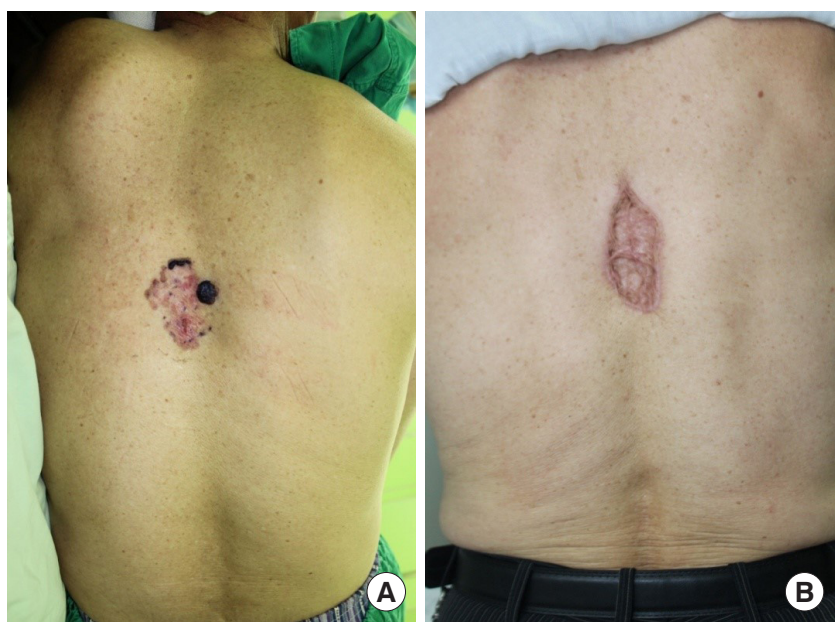

Fig. 1. Squamous cell carcinoma in situ on the back. (A) A $5 \times 5.5 \mathrm{~cm}$ area of skin discoloration with protruding masses on the back. Local excision of the protruding masses was performed, and a histopathological examination revealed squamous cell carcinoma in situ with a positive resection margin. (B) Photograph taken 7 months after additional wide excision and split-thickness skin grafting.
STSGs. Histopathology confirmed BCC on the neck with a positive resection margin. Based on histopathological findings, five lesions on the right forearm and hand were diagnosed as SCC in situ, and a positive resection margin was observed at two sites. Repeated excisions were performed on the neck and the right forearm, where the resection margin was positive. The resection margin was then confirmed to be negative. All wounds healed without complications.

The patient visited an otorhinolaryngology clinic for a sore throat 1 year after presentation to our clinic and was diagnosed with supraglottic SCC and SCC in the external auditory canal. An otolaryngologist at another hospital performed a supraglottic partial laryngectomy with selective neck dissection at levels 2-4 for the supraglottic SCC, and lateral temporal bone resection with superficial parotidectomy for the SCC in the external auditory canal. Adjuvant chemotherapy was also performed.

Two years after the patient's initial presentation at our clinic, he presented again with multiple pigmented lesions of the neck and the helices of both ears. All the lesions were excised and confirmed as SCC in situ with clean margins through a histopathological examination. The neck lesion recurred, and additional resection was performed. Wide excision was simultaneously performed for multiple pigmented lesions of the dorsum of the left hand. All these lesions were diagnosed as SCC in situ (Fig. 3). Four months later, four pigmented lesions were observed on the right pre-auricle, temporal scalp, and temple. All these lesions were diagnosed as SCC in situ, with clear margins on biopsy.
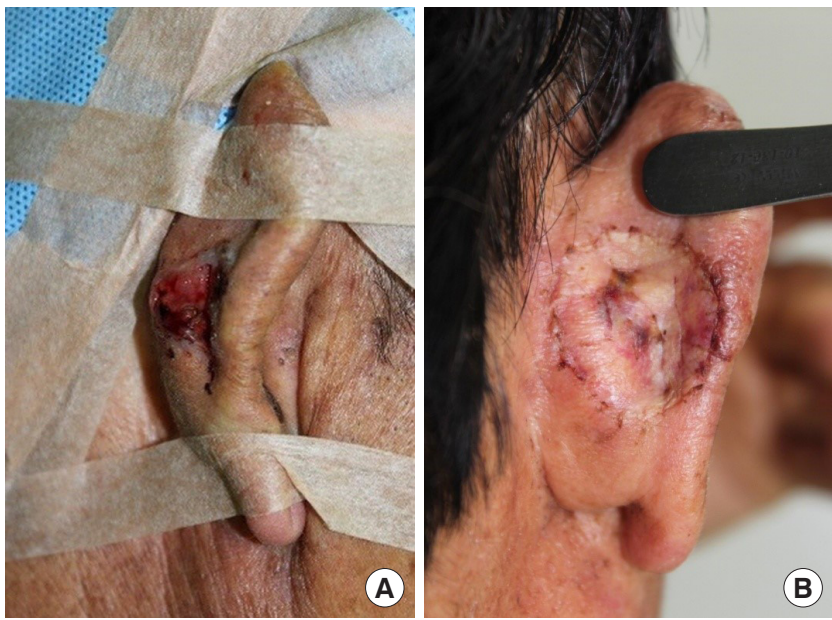

Fig. 2. Basal cell carcinoma of the retro-auricle. (A) Preoperative photograph of a $1.5 \times 1.5 \mathrm{~cm}$ ulcer on the right retro-auricle that was diagnosed as basal cell carcinoma based on a histological examination; subsequently, wide excision and split-thickness skin grafting were performed. (B) Two-week postoperative photograph. 

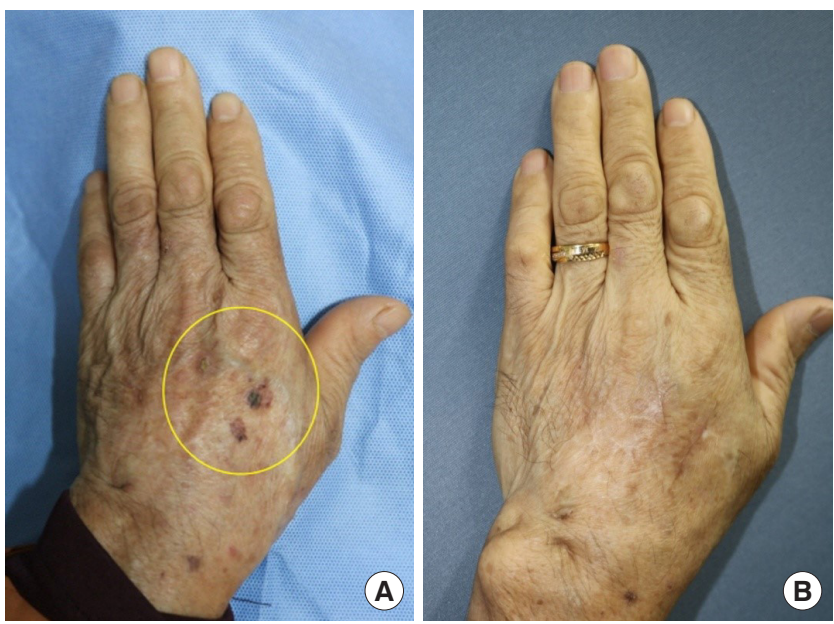

Fig. 3. Squamous cell carcinoma in situ on the hand. (A) Pigmented lesions on the dorsum of the left hand. Wide excision and direct closure were performed. (B) Three-month postoperative photograph.

The patient presented with several pigmented lesions on his right ear in the following year. Incisional biopsy was performed for the lesions in the triangular fossa, while excisional biopsy was performed for the lesions on the pre-auricle and helix. The lesion in the triangular fossa was confirmed to be SCC in situ. The preauricular lesions were confirmed as micro-invasive SCC with a clear resection margin and no lymphovascular invasion. Wide excision and a full-thickness skin graft were performed for the SCC in situ in the triangular fossa of the patient's right ear. All wounds healed without complications (Fig. 4).

\section{DISCUSSION}

Ultraviolet (UV) radiation is the best-known and most significant risk factor for NMSC. Other factors associated with BCC include a family history of skin cancer, old age, male sex, exposure to arsenic and dry ice, a high-fat diet, and Fitzpatrick skin types 1 and 2 [7]. The risk factors for SCC are similar, and include fair skin, a poor ability to tan, a history of blistering by sunburns, a family history of SCC, a diagnosis of HPV, outdoor jobs, habitual smoking, and numerous psoralen and UV-A treatments [8]. Our patient had no family history of skin cancer, had Fitzpatrick skin type 4, and did not have any relevant risk factors, including a history of smoking. Furthermore, he was never exposed to arsenic, had never received psoralen or UV-A treatment, and had no history of HPV infection. The patient's risk factors were age (67 years old at the time of his first diagnosis at our hospital), sex (male), and occupation (farmer).

Most BCCs have only local sequelae and do not lead to death or metastasis. However, because SCCs can metastasize to other organs, high-risk patients should be monitored carefully. The
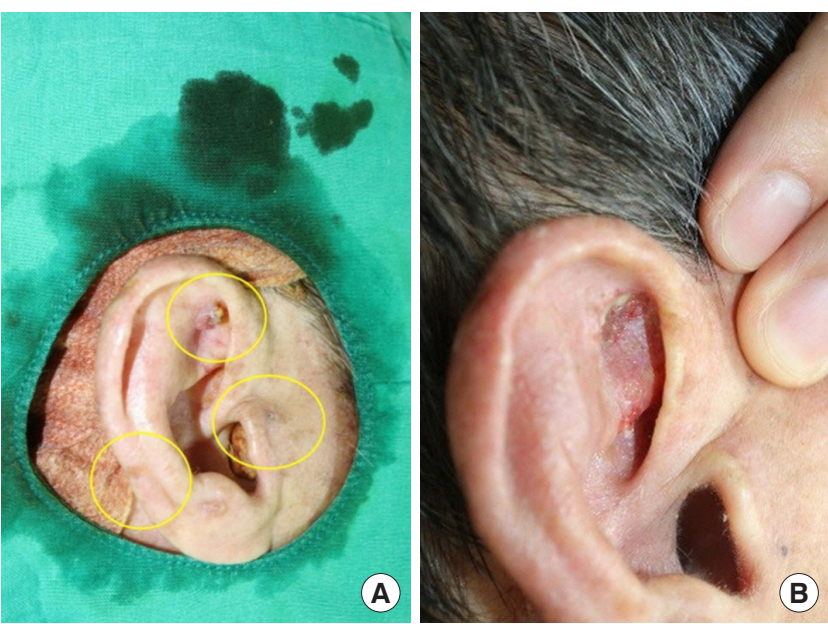

Fig. 4. Multiple squamous cell carcinomas of the ear. (A) Multiple masses on the right ear and pre-auricle. The preauricular lesions were confirmed to be micro-invasive squamous cell carcinoma. Based on a histopathological examination, the triangular fossa lesion was diagnosed as squamous cell carcinoma in situ. Wide excision and full-thickness skin grafting were performed. (B) On the eighth day after skin grafting, minor disruption was observed, but epithelization was complete with secondary intention.

factors specified by the American Joint Committee on Cancer as being associated with a high risk of SCC are a tumor thickness of $>2 \mathrm{~mm}$ and a Clark level of IV or greater, perineural invasion, location of the tumor on the ear or a non-hair-bearing lip, and poor differentiation or lack of differentiation of the tumor. According to Brigham and Women's Hospital, the factors associated with high risk are a tumor diameter of $\geq 2 \mathrm{~cm}$, poor differentiation of tumor, perineural invasion of $\geq 1 \mathrm{~mm}$, and tumor invasion beyond the fat (excluding bone invasion, which would upgrade the tumor to stage T3). A previous study also reported that the depth of invasion, histological findings, cancer location, horizontal size, perineural invasion, recurrence, incomplete excision, the presence of multiple cancers, genetic or molecular factors, and patient-related characteristics could increase the risk of metastasis [9]. Because the patient described herein was deemed to be high-risk due to the tumors' location and horizontal diameter, recurrence, and the presence of multiple cancers, a contrast-enhanced computed tomography evaluation was performed, but no evidence of metastasis was found on imaging.

In a 10-year prospective study conducted in Australia, over two-thirds of patients with NMSC developed additional skin cancer within 10 years. The main risk factor for new skin cancer was the number of previously diagnosed skin cancers, while male sex was a secondary risk factor [10]. According to a single cohort study comparing patients with one CSCC to those with multiple CSCCs, a higher number of skin cancers (especially 10 
or more CSCCs) was associated with an increased likelihood of recurrence, lymph node metastasis, and poor prognosis. Approximately $80 \%$ of the patients with 10 or more CSCCs were immunosuppressed [11]. Marcil and Stern [12] conducted a literature review and meta-analysis to evaluate the occurrence of subsequent NMSC according to the type of the initial NMSC. The 3-year cumulative risk of developing subsequent SCC after the first occurrence of SCC was 18\%, at least 10 times higher than that observed in the general population. The 3-year cumulative risk of subsequent BCC was $44 \%$, which was also more than 10 times higher than that observed in the general population. Patients with a history of SCC had an equivalent risk of developing BCC to patients with a history of BCC, whereas patients with a history of BCC had a lower risk of developing SCC than those with a history of SCC [12]. In the patient described herein, numerous NMSCs and more than 10 CSCCs were diagnosed, and new skin cancers continued to occur. The patient was old and had a history of chemotherapy after otolaryngologic surgery. Patients with these characteristics require careful observations of scar sites and lymph node examinations. In addition to NMSC, melanoma can also occur in patients with multiple skin cancers. Therefore, in patients with multiple NMSCs, periodic follow-up is necessary because their risk of additional NMSC and melanoma is higher than that of the normal population [10].

Squamous cell carcinoma antigen (SCCA) is most commonly used as a biomarker of cervical cancer, but also serves as a tumor marker of SCCs more broadly, including those in the head and neck area [13]. In our case, SCCA was within the normal range. Limited research has evaluated the usefulness of biomarkers for skin cancer. Future studies should investigate biomarkers for skin cancer in order to improve the accuracy of patients' prognosis and to facilitate early diagnosis.

This patient was diagnosed with supraglottic SCC and CSCC and underwent surgical treatment. Laryngeal cancer is one of the most common cancers of the head and neck area, and its major risk factors are heavy smoking and alcohol consumption [14]. The symptoms of laryngeal cancer include nonspecific throat pain, dysphagia, and cervical lymphadenopathy. SCC in the head and neck area mostly metastasizes to the lungs (70\%$75 \%)$, liver (17\%-38\%), and bones $(23 \%-44 \%)$ through the hematogenous route [15]. However, skin metastasis has been reported in rare cases (1\%-2\%) [16]. In our case, all lesions were diagnosed as primary SCC, and no metastatic lesions were observed on positron emission tomography.

Saini et al. [17] reported multiple NMSC lesions on a patient's head and trunk. Although the literature on multiple skin cancers is relatively abundant, our case seems very rare, as the pa- tient developed multiple types of skin cancer in several locations over many years. Furthermore, it is very unusual for primary SCC to develop throughout the body, including the head and neck, as occurred in our case.

In addition to the lesions confirmed as NMSC, biopsies were performed on numerous suspected lesions. Histopathological examinations confirmed that most of these lesions were actinic keratosis (AK). Some lesions diagnosed as SCC in situ coexisted with $\mathrm{AK}$, which is a disease continuum that progresses to SCC. The risk factors for AK are old age, sun exposure, and immunosuppression, similar to those for NMSC. When AK progresses to SCC, changes such as ulceration, redness, and growth occur, but biopsy is the only way to distinguish between these two conditions [18]. Therefore, skin lesions that could be cancerous, even if they appear benign, should be biopsied.

\section{NOTES}

\section{Conflict of interest}

No potential conflict of interest relevant to this article was reported.

\section{Ethical approval}

The study was approved by the Institutional Review Board of Konkuk University Chungju Hospital (IRB No. KUCH 201911-037) and performed in accordance with the principles of the Declaration of Helsinki. Written informed consent was obtained.

\section{Patient consent}

The patient provided written informed consent for the publication and the use of his images.

\section{ORCID}

Song Hyun Han https://orcid.org/0000-0002-2726-2583

Soon Heum Kim https://orcid.org/0000-0001-9773-4753

Cheol Keun Kim https://orcid.org/0000-0003-2126-9749

Dong In Jo https://orcid.org/0000-0002-3075-4482

\section{REFERENCES}

1. Rogers HW, Weinstock MA, Feldman SR, Coldiron BM. Incidence estimate of nonmelanoma skin cancer (keratinocyte carcinomas) in the U.S. population, 2012. JAMA Dermatol 2015; 151:1081-6

2. Motley R, Kersey P, Lawrence C; British Association of Dermatologists; British Association of Plastic Surgeons; Royal College of Radiologists, Faculty of Clinical Oncology. Multiprofessional 
guidelines for the management of the patient with primary cutaneous squamous cell carcinoma. Br J Dermatol 2002;146:1825.

3. Veness MJ. High-risk cutaneous squamous cell carcinoma of the head and neck. J Biomed Biotechnol 2007;2007:80572.

4. Nakayama M, Tabuchi K, Nakamura Y, Hara A. Basal cell carcinoma of the head and neck. J Skin Cancer 2011;2011:496910.

5. Chockalingam R, Downing C, Tyring SK. Cutaneous squamous cell carcinomas in organ transplant recipients. J Clin Med 2015;4:1229-39.

6. Chuang TY, Popescu NA, Su WP, Chute CG. Squamous cell carcinoma: a population-based incidence study in Rochester, Minn. Arch Dermatol 1990;126:185-8.

7. Kim DH, Ko HS, Jun YJ. Nonsyndromic multiple basal cell carcinomas. Arch Craniofac Surg 2017;18:191-6.

8. Lorimer P, Milas Z. Cutaneous manifestations associated with malignancy of the head and neck. Semin Oncol 2016;43:353-8.

9. Burton KA, Ashack KA, Khachemoune A. Cutaneous squamous cell carcinoma: a review of high-risk and metastatic disease. Am J Clin Dermatol 2016;17:491-508.

10. Czarnecki D, Sutton T, Czarnecki C, Culjak G. A 10-year prospective study of patients with skin cancer. J Cutan Med Surg 2002;6:427-9.

11. Levine DE, Karia PS, Schmults CD. Outcomes of patients with multiple cutaneous squamous cell carcinomas: a 10-year sin- gle-institution cohort study. JAMA Dermatol 2015;151:1220-5.

12. Marcil I, Stern RS. Risk of developing a subsequent nonmelanoma skin cancer in patients with a history of nonmelanoma skin cancer: a critical review of the literature and meta-analysis. Arch Dermatol 2000;136:1524-30.

13. Izuhara K, Yamaguchi Y, Ohta S, Nunomura S, Nanri Y, Azuma Y, et al. Squamous cell carcinoma antigen 2 (SCCA2, SERPINB4): an emerging biomarker for skin inflammatory diseases. Int J Mol Sci 2018;19:1102.

14. Peller M, Katalinic A, Wollenberg B, Teudt IU, Meyer JE. Epidemiology of laryngeal carcinoma in Germany, 1998-2011. Eur Arch Otorhinolaryngol 2016;273:1481-7.

15. Calhoun KH, Fulmer P, Weiss R, Hokanson JA. Distant metastases from head and neck squamous cell carcinomas. Laryngoscope 1994;104:1199-205.

16. Trehan R, Pandey AK, Khosla D, Dimri K, Punia RS. Apropos of a case of cutaneous metastasis from laryngeal cancer with review of literature. J Cancer Res Ther 2015;11:655.

17. Saini R, Sharma N, Pandey K, Puri KJ. Multiple skin cancers in a single patient: multiple pigmented Bowen's disease, giant basal cell carcinoma, squamous cell carcinoma. J Cancer Res Ther 2015;11:669.

18. Moy RL. Clinical presentation of actinic keratoses and squamous cell carcinoma. J Am Acad Dermatol 2000;42(1 Pt 2):810. 